\title{
Research on Multi-Fault Phased Rush Repair Strategy of Distribution Networks
}

\author{
Zheliang Li ${ }^{1,2, a}$, Pingping Bai ${ }^{1,2, b}$, Qiulu Wang ${ }^{\text {3, c }}$, Mu Zhu ${ }^{4, d}$ \\ ${ }^{1}$ State Grid Henan Electric Power Company Luoyang Power Supply Company, \\ Luoyang 471000, China \\ ${ }^{2}$ School of Electrical Engineering, Wuhan University, Wuhan 430072, China \\ ${ }^{3}$ Luoyang City Planning and Architectural Design \& Research Institute Company. Luoyang 471000 , \\ China \\ ${ }^{4}$ State Grid Henan Electric Power Company Maintenance Company, Zhengzhou 450000, China \\ a935769881@qq.com, b1015903240@qq.com
}

Keywords: Mutil-fault, rush repair, distribution networks

\begin{abstract}
The distribution network is complicated, it often causes many faults when happing natural disasters. Relying on the repair dispatching experience, it is difficult to develop the most effective repair scheme. In view of the feature that the rush repair faults after the disaster is different from the general. It emphasizes the repair fast and timeliness, as if it can't recover the power fast, and it is the prerequisite of the disaster repair. Therefore it is important to develop a emergency platform of optimal repair scheduling with limited repair persons and resources, improving the post-disaster grid power supply security.
\end{abstract}

\section{Introduction}

As the end of the electricity distribution network system, and the normal operation of the enterprise and is closely related to people's lives, their security and stable operation of the production is the basis of today's social and economic progress. With the rapid development of science and technology, smart meters and smart appliances and other power distribution equipment which is widely used in the power grid, power grid construction gradually strengthen, upgrade, network structure more complex, so close to the limit distribution network gradually stable operation. Country prone is natural disasters. In addition to natural disasters caused losses of life and property of the people outside, the grid will be destroyed, while electricity supply is essential repairs after the disaster, if the power supply cannot be quickly restored, the direct impact on the smooth progress of other relief efforts. Such examples abound, 2008 South snowstorm, a large tower collapsed, the line was crushed, causing widespread loss of electricity, its long duration, high-impact area of the subject, it is rare in the history of our country, to the people production and life severely affected; earthquake ensued is on Power emergency rescue major test, in addition, Gansu Zhouqu earthquake, 2010 continuous typhoons and other disasters to us sounded the alarm.

Disasters occur due to multiple distribution system equipment failure, the load (especially important load) loss of power, an important sector for hospitals, government, such as the power supply cannot be quickly restored, losses cannot be estimated, and therefore need to quickly repair the fault . The disaster of the fault repair is different from the general failure to fix the problem, its greater emphasis on rapid repair, timeliness, such as the destruction of the power grid, the power supply cannot be obtained, it will affect other disaster relief efforts, and the rescue work is implemented in other disaster premise and guarantee. Understanding power facilities damaged condition, rational and efficient organization of repair; you can enhance the efficiency of disaster contingency and reduce all kinds of losses and due to power outages caused by the impact. So, how in the limited repair personnel and resources, to develop a scientific and efficient repair strategies become a matter of growing concern. Repair strategy is to develop fast repairs to restore power after the disaster the core of the research has important significance. 
Although the different distribution network and transmission network structure, bound by different conditions in the development of repair strategies, different considerations, but the implementation depends on the artificial intelligence, artificial intelligence optimization choose to give a final repair strategies, generate repair program. Taking into account the grid fault repair of complexity and uncertainty, and the distribution network is relatively simple, and it prone to failure scenarios in real life, and people's lives, while easy to build a mathematical model, the constraints are easy to quantify, Therefore, this paper will be targeted distribution network, the distribution network fault repair disaster more in-depth study.

\section{Optimization Algorithm for Fault Repair of Distribution Network}

The distribution network fault repair more simplified mathematical model, that is a point of failure and reverse brake repair order to control the switching operation of the multi-variable constrained nonlinear mixed integer multi-objective optimization problem. Complex distribution network topology, many branches, after the disaster, fault repair, and switching operation Too many variables, there is often a large amount of data to calculate the slow and so on. In this regard, domestic and foreign scholars have done a lot of research, proposed several ways to solve this problem, summed up into two categories: traditional optimization algorithms and artificial intelligence methods.

(1)Traditional optimization algorithms. Traditional optimization algorithms are used to calculate the repair teams to repair the fault point sequentially through a variety of mathematical optimization techniques, draw final restore power strategy, which will be expressed as objective optimization problem solving functions and equations using standard mathematical form, inequality constraints, the use of higher mathematics to solve differential thinking to obtain the final optimal solution. Traditional optimization algorithm is a major advantage is that when there is a problem to be solved when the optimal solution, by which it will certainly be calculated, for some relatively simple optimization problem solving in general to meet the requirements, but for which actual failure points more, with complex nonlinear grid, high-dimensional problems, often to optimize a long time, and often not the best solution, not even to know the approximate extent of the resulting solution with optimal solutions.

(2) In recent years, many scholars by the nature of biological phenomena inspired food, made a number of artificial intelligence optimization methods in power system fault repair them to get more and more applications, the main method of expert system (Expert System), heuristic algorithm, genetic algorithm (Genetic Algorithm, GA), fuzzy algorithm (Fuzzy Algorithm, FA), Petri net algorithms, PSO (Particle Swarm Optimization Algorithm, PSO), bacterial colony chemotaxis algorithm (Bacterial Colony Chemotaxis Algorithm, DBCC) and so on.

\section{DG's Distribution Network Containing Multiple Fault Repair Phased Strategy}

In recent years, distributed generation technology has developed rapidly, gaining more and more attention, coordination and complementarily of its power grid, to provide users with reliable, high-quality energy, but also for the development of post-failure repair strategies offer much more choice. Meanwhile, to improve repair efficiency, part of the power company is equipped with emergency vehicles and other emergency power supply, with the emergency power supply, flexible dispatch characteristics, the role of the car in the repair of power generation becomes more obvious, the two complement each other to ensure that important after failure load quickly restore power, which greatly reduces the social and economic losses. Based on this, the chapter after the loss of power distribution network based on priority to restore important load power supply situation, the economy and repair time on both sides, a priority to restore a load with the secondary load, the establishment of a distribution under the condition of distributed generation Optimization Model for network disaster more fazed repair team. Repair process, in addition to the use of contact switch switching operation with repairs, taking into account DG islanding operation, and emergency vehicles as an important influence on the load power supply and other temporary repair, improve 
repair efficiency. It improved optimization algorithm to quickly get repair strategies using DBCC, and finally the simulation to validate the model and the proposed method of distribution network 69 via bus system.

After the distribution network failure, loss of power load and each load level and the capacity of different sizes, load priority should be to ensure that important repairs to restore power, which is consistent with the actual situation of the distribution network. Based on this paper establishes a phased repair model, the first stage is important to ensure fast load short supply, economy and timeliness from two considerations, the establishment minimize economic losses and repair the shortest time multi-objective optimization model; the second phase the remaining three load recovery, then the repair time is not a priority repair factors, only for the goal to minimize economic losses to optimize repair.

Based distribution network radiating characteristics, proposed efficient search algorithm network topology information and determine node fitness evaluation. In the first phase of the repair process, for example for illustration: (1) Enter the initial network topology data (node number, type, load node information, switch status, repair estimated between standard time, node drive time, emergency generator car capacity, etc.). (2) According to the text of the coding principles of bacterial sequences obtained by coding repair, according to the encoded information distribution and repair tasks for the team, dispatch emergency vehicles to load node power supply that matches the capacity of simultaneously recorded time. (3) DG splitting operation, the judge DG capacity, if they can form a multi-user island operation, the splitting generated multiuser islands, recording operating time; if multiuser DG insufficient capacity margin, direct solution silos as a plurality of units; DG islands within the cell capacity is less than the load carried by the capacity to determine whether the conduct load shedding operation, can, DG islanding after load shedding, power supply time recording, DG otherwise disconnected from the grid power supply is stopped. (4) Determination of the node type. The actual fault point determination node or virtual point of failure, and then search for the fault on both sides by a point, the lower charge states on judgment: live side point of failure, determine the type of power charged side. If the power supply network, the number of search energized side electric load loss, economic loss computing device fault eigenvalues $\lambda$, repair and modify the connection between the fault and charging status; if DG supply, fault repair, and modify the network connection between the charged state, DG and network operation over the same period. (5) The location and repair time repair team calculations. As for the failed node, the repair team location to the current node, and the cumulative drive time and fault repair time; such as a virtual point of failure, repair teams to maintain the original position of the same node, the time will not be accumulated. (6) the charged state of the network node to determine whether all restore lost power load power supply, if not fully recovered then go to step (2), if all loads are to restore power to obtain socio-economic losses and repair time.

The establishment of a distribution network disaster staged repair policy model with DG sudden case of multiple failures, considering more team synergy repair, make full use of the model DG generating capacity to ensure rapid supply critical loads, load and two-level priority restoration level load power supply, taking into account the contact switch switching operation and dispatch emergency vehicles with repair, improve repair efficiency. Improved BCC discrete optimization algorithm optimization model, it gets repair strategy quickly. Simulation example shows that the model can effectively improve repair efficiency and reduce socio-economic losses. After all the fault repair is completed, the distribution system due to the switching operation of the switch contact may be in a non-operational state of the economy, it can be network reconfiguration under conditions permitting, to make the distribution network restored to its original operating mode. 


\section{Summary}

Fault dispatcher came to develop empirically fault repair order to meet repair fast, real-time requirements, and once a major natural disaster, many device failure occurs, large amounts of information in a short transfer to SCADA (Supervisory Control And Data Acquisition) systems, relying on experience dispatcher does not give a reasonable repair strategy, then how that information analyzed with a choice of an excellent intelligence optimization algorithm for the repair team to develop effective fault repair program, is a problem worthy of study. Distribution network for disaster multiple faults quickly repair the problem, the paper much fault repair disaster model, multi-agent system implementation done in-depth research, has achieved the following results: presents a chemotaxis algorithm based on improved bacterial population distribution network disaster much fault repair strategy. BCC algorithm is applied to the improved distribution network more fault repair them, define the center as well as targeted mutation chemotactic factor of two improvement, to solve high-dimensional distribution network, the amount of data caused by the convergence rate is slow, easy to fall into local optimum problems.

\section{Reference}

[1]Yang Lijun, Zhang Jing, Cheng Huilin, et al. A Task Allocation Strategy of Multi-fault Rush Repair for Distribution Network Based on Optimum Utility[J]. Transactions of China Electrotechnical Society, 2014.

[2]Zhang J, Zhang L, Huang X. A Multi-fault Rush Repair Strategy for Distribution Network Based on Genetic-topology Algorithm[J]. Automation of Electric Power Systems, 2008.

[3]Zhigang L U, Sun B, Liu Z, et al. A Rush Repair Strategy For Distribution Networks Based On Improved Discrete Multi-Objective Bcc Algorithm After Discretization[J]. Dianli Xitong Zidonghua/automation of Electric Power Systems, 2011, 35(11):55-59.

[4]Li X, Lu Z, Liu Z, et al. Multi-agent Strategy of Distribution Networks Multi-Faults Rush-Repair with Distributed Generators[J]. Transactions of China Electrotechnical Society, 2013, 28(8):48-55.

[5]Zhigang L U, Zhige Y E, Yang L. An Approach of Time Interval-Divided Multi-Fault Dynamic Restoration for Distribution Network Based on Blackboard Model[J]. Power System Technology, 2012, 36(9):198-202. 